Bundesgesundheitsbl 2019 $\cdot 62: 84-93$ https://doi.org/10.1007/s00103-018-2854-4 Online publiziert: 29. November 2018 (c) Der/die Autor(en) 2018

CrossMark

\author{
Diana Kurch-Bek' · Christian Gallowitz' • Bernhard Tenckhoff' • \\ Leonie Sundmacher ${ }^{2}$ \\ 'Innovation, strategische Analyse und IT-Beratung, Kassenärztliche Bundesvereinigung, Berlin, \\ Deutschland \\ ${ }^{2}$ Fachbereich Health Services Management, Ludwig-Maximilians-Universität München, München, \\ Deutschland
}

\title{
Influenzaimpfquoten von Patienten mit vertragsärztlicher Versorgung in beschützenden Einrichtungen, Pflege- und Altenheimen
}

\section{Hintergrund und Fragestellung}

Influenzaausbrüche in beschützenden Einrichtungen sowie Pflege- und Altenheimen (kurz: bPA-Einrichtungen) betreffen in besonderem Maße ältere oder multimorbide Menschen, die ein erhöhtes Risiko für schwerwiegende Krankheitsverläufe tragen. Jährlich treten in den Wintermonaten Grippewellen mit unterschiedlicher Intensität auf. Saisonale Influenzainfektionen gehen mit einer Vielzahl an Erkrankungsund Todesfällen sowie Krankenhauseinweisungen einher und stellen für die öffentliche Gesundheitsversorgung eine bedeutende Infektionskrankheit mit hohen Kosten dar [1-3]. Die kosteneffektivste Präventionsmaßnahme gegen eine Influenzaerkrankung ist die Impfung, die jährlich vor Beginn der Influenzasaison durchgeführt werden sollte. Neben dem individuellen, gesundheitlichen Nutzen von Impfungen für Einzelne ist die bevölkerungsweite Effektivität abhängig von der Durchimpfung. Durch hohe Impfquoten erhöht sich der gesundheitliche und ökonomische Nutzen, da eine Senkung der Krankheitslast und Verlängerung der ausbruchsfreien Zeiträume zu erwarten ist $[4,5]$. Die ständige Impfkommission (STIKO) empfiehlt eine Influenzaimmunisierung für Kinder, Jugendliche und
Erwachsene mit erhöhter gesundheitlicher Gefährdung durch Grundleiden oder mit beruflicher Exposition sowie für Personen ab 60 Jahren und insbesondere für Bewohner von Alters- oder Pflegeheimen. Seit der Saison 2014/2015 fordert die Europäische Kommission aufgrund der hohen Krankheitslast eine Influenzaimpfquote von $75 \%$ für die ältere Bevölkerung [6].

Influenzaimpfquoten werden im Rahmen von nationalen und internationalen Qualitätsindikatoren erfasst. Insbesondere wird über Patienten mit chronischen Erkrankungen berichtet [7-10]. In Deutschland liegen bereits bundesweite Studien zu Impfquoten bei Erwachsenen und über 60-Jährigen zur Umsetzung der Influenzaempfehlung auf Basis von Routinedaten vor, welche Hinweise auf regionale Unterschiede in der Inanspruchnahme aufzeigen $[11,12]$. Bödeker et al. geben einen ersten Einblick in die Umsetzung der Influenzaempfehlungen in Alten- und Pflegeheimen. Sie kommen zu dem Schluss, dass Studien zu Influenzaimpfquoten fehlen sowie zu relevanten Faktoren, welche die Impfentscheidung der Bewohner von Alten- und Pflegeheimen beeinflussen [13]. Ferner fanden sich in Studien Hinweise auf die Rolle der Hausärzte zur Motivation bei Impfungen [13-18]. Für Patienten in Alten- und Pflegeheimen zeigen verschie- dene internationale Studien erreichbare Impfquoten zwischen $81 \%$ und $93 \%$ $[19,20]$. In Deutschland existieren jedoch keine aussagekräftigen Analysen zu den Influenzaimpfquoten von Bewohnern in bPA-Einrichtungen. Diese Lücke soll mit der vorliegenden Analyse auf Basis von Routinedaten verkleinert werden.

Ziel dieser Arbeit ist es, Daten über die deutschlandweiten Impfquoten zur Influenza von in bPA-Einrichtungen vertragsärztlich versorgten Patienten im zeitlichen Verlauf der letzten 6 Impfsaisons (2010/2011 bis 2015/2016) darzustellen und zu untersuchen. Die Impfquoten können erste Hinweise auf die Umsetzung der Empfehlungen der STIKO und der Europäischen Kommission geben [6, 8].

\section{Methoden}

\section{Datengrundlage}

Datengrundlage sind die bundesweiten ambulanten vertragsärztlichen Abrechnungsdaten der Jahre 2010 bis 2016. Diese enthalten Informationen über abgerechnete Diagnosen gemäß ICD-10 GM (International Statistical Classification of Diseases and Related Health Problems 10 German Modification; [21]) und Leistungen auf Basis des Einheitlichen Bewertungsmaßstabes (EBM). Im 
EBM ist jeder Leistung eine Nummer, die sogenannte Gebührenordnungsposition (GOP), zugeordnet [22]. Der EBM spezifiziert die Abrechnung und Vergütung von vertragsärztlichen Leistungen. Ergänzend können Kassenärztliche Vereinigungen (KVen) spezifische Leistungen (kurz: KV-spezifische GOPen) aufnehmen, welche durch gesonderte Verträge vereinbart wurden (z.B. Hausbesuche in Pflegeheimen, Impfleistungen). In dieser Datengrundlage ist jede in der gesetzlichen Krankenversicherung (GKV) versicherte Person mit mindestens einem Vertragsarztkontakt im entsprechenden Abrechnungszeitraum erfasst.

\section{Abbildung der Influenza- impfsaisons}

Zu Beginn einer Influenzasaison wird eine jeweils angepasste Influenzaimpfung empfohlen. Erste Impfungen können ab September erfolgen, empfohlen wird die Impfung ab der 40. Kalenderwoche. Im Rahmen der ambulanten Versorgung erfolgen die Impfungen im Zeitraum zwischen dem 3. Quartal und dem 1. Quartal des Folgejahres. In der vorliegenden Analyse wurden die einzelnen Impfsaisons von 2010/2011 (3. Quartal 2010 bis 1. Quartal 2011) bis 2015/2016 (3. Quartal 2015 bis 1. Quartal 2016) betrachtet.

\section{Bildung von Studienkohorten und Berechnung der Impfquoten}

In den Basispopulationen der jeweiligen Saison wurden alle gesetzlich krankenversicherten Patienten erfasst, die im Untersuchungsjahr zu Beginn der Impfsaison mindestens einen Arztkontakt hatten. Es wurden lediglich Patienten berücksichtigt, deren Geschlecht sowie Geburtsjahr eindeutig bestimmbar waren. Um die Gruppe der Patienten in bPAEinrichtungen adäquat abzugrenzen, erfolgte der Einschluss von Patienten ab 60 Jahren bis 110 Jahren [22, 23]. Aus den Basispopulationen wurde in der vorliegenden Studie für jedes Jahr im Analysezeitraum eine Studienkohorte wie folgt gebildet (• Abb. 1): Zur Operationalisierung von Patienten in bPA-Einrichtungen wurde sowohl die bundeseinheitliche GOP 01415 (im Folgenden: Besuchs- pauschale) herangezogen, welche sich auf den dringenden Besuch oder Besuch in einem beschützenden Wohnheim bzw. Einrichtung bzw. Pflege- oder Altenheim mit Pflegepersonal bezieht, als auch KVspezifische GOPen. Es wurden Patienten erfasst, ausgehend vom ersten bis vierten Quartal eines Jahres, für die in dem Zeitraum die Besuchspauschale oder eine $\mathrm{KV}$-spezifische GOP mindestens 2-mal abgerechnet wurde (M2Q: in mindestens 2 Quartalen). Durch die Anwendung des M2Q-Kriteriums konnten die dauerhaft in bPA-Einrichtungen lebenden Patienten gegenüber Patienten der Kurzzeitpflege und Patienten, die kurzfristig nach dem Übergang in einer bPA-Einrichtung versterben, abgegrenzt werden. Ausgehend von den Patienten in bPA-Einrichtungen mit Inanspruchnahme der vertragsärztlichen Versorgung im Kalenderjahr (z. B. 2010) wurden nur Patienten mit mindestens einer beliebigen Leistungsinanspruchnahme in der jeweiligen Impfsaison (z. B. 3. Quartal 2010 bis 1. Quartal 2011) eingeschlossen, um die Patienten in bPA-Einrichtungen exakt abzugrenzen beispielsweise gegenüber verstorbenen Patienten. A Abb. 1 stellt die Bildung der Studienkohorten der Impfsaisons im Flussdiagramm dar.

Die regionale Zuordnung eines Patienten erfolgte nach dem Wohnortprinzip (letzter Wohnort im jeweiligen Beobachtungszeitraum einer Impfsaison) und wurde auf der Ebene der KVen, welche mit Ausnahme von Nordrhein-Westfalen den Bundesländern entspricht, sowie getrennt nach alten und neuen Bundesländern ausgewertet. Darüber hinaus erfolgte die Einteilung in 3 Kreistypen: Kernstädte, verdichtetes Umland und ländliches Umland [24]. Um die hausärztliche Versorgung abzugrenzen und näher zu untersuchen, wurden die im Rahmen der Abrechnungsstatistik der Kassenärztlichen Bundesvereinigung benutzten Abrechnungsgruppenzuordnungen verwendet. Zur weiterführenden Analyse der Impfsaisons wurden zusätzlich Patienten in bPA-Einrichtungen als chronisch krank markiert, wenn eine gesicherte Diagnose mindestens 2-mal codiert wurde (M2Q je Impfsaison). Als Grundlage diente die von Riens et al. verwendete Definition von chronischen
Erkrankungen auf Basis des ICD-10 GM, mit Ausnahme der transitorischen ischämischen Attacke, da diese ein akutes Krankheitsbild darstellt [11, 21].

Zur Berechnung der Impfquote wurde der Anteil der Patienten mit abgerechneter GOP für eine Influenzaimpfung bezogen auf die Anzahl der Patienten in der jeweiligen Studienkohorte gebildet. Darüber hinaus wurden die Impfquoten stratifiziert nach Chronikern, Geschlecht, Altersgruppen oder regionaler Zuordnung berechnet und dargestellt $[22,24]$.

Die Validierung der Studienkohorte (Übersicht über die Korrespondenzadresse erhältlich) erfolgte anhand der Anzahl verfügbarer vollstationärer Plätze in Pflegeheimen, welche in der Gesundheitsberichterstattung des Bundes (Pflegestatistik) bereitgestellt wird [25].

\section{Statistische Analyse}

Die statistischen Analysen wurden mit R (Version 3.2.5) durchgeführt. Für regionale Vergleiche der Influenzaimpfung wurde die relative Häufigkeit der gegen Influenza geimpften, in bPA-Einrichtungen vertragsärztlich versorgten Patienten $\mathrm{zu}$ allen in bPA-Einrichtungen vertragsärztlich Versorgten dargestellt.

\section{Ergebnisse}

\section{Basis- und Studienpopulationen}

Über die Basispopulation der Jahre waren durchschnittlich 28,6\% der Patienten mindestens 60 Jahre und höchstens 110 Jahre alt. Drei von hundert über 60jährige Patienten wiesen eine mindestens in 2 Quartalen abgerechnete Besuchspauschale oder KV-spezifische GOP auf, welche sich auf einen Vertragsarztkontakt in einer bPA-Einrichtung bezog. Als vertragsärztlich versorgte bPA-Patienten konnten in der ersten Impfsaison 461.941 und in der letzten betrachteten Impfsaison 462.028 Personen identifiziert werden. Aus diesen Patienten ergeben sich die Studienkohorten (Grundgesamtheit) für die Impfsaisons (• Tab. 1). In der Gesundheitsberichterstattung des Bundes werden die verfügbaren vollstationären Plätze in Pflegeheimen für die Jahre $2011(N=830.781), 2013(N=847.705)$ 
Bundesgesundheitsbl 2019 · 62:84-93 https://doi.org/10.1007/s00103-018-2854-4

(c) Der/die Autor(en) 2018

D. Kurch-Bek · C. Gallowitz · B. Tenckhoff · L. Sundmacher

\section{Influenzaimpfquoten von Patienten mit vertragsärztlicher Versorgung in beschützenden Einrichtungen, Pflege- und Altenheimen}

\section{Zusammenfassung}

Hintergrund. Eine der wichtigsten und wirksamsten Präventionsmaßnahmen zum Schutz vor saisonaler Influenza sind Impfungen. Die ständige Impfkommission (STIKO) empfiehlt die Influenzaimpfung für besondere Personengruppen, wie Patienten in beschützenden Einrichtungen sowie in Pflegeund Altenheimen (bPA-Einrichtungen). Die Europäische Kommission (EK) fordert eine Influenzaimpfquote von $75 \%$ in der älteren Bevölkerung. In Deutschland liegen bisher nur vereinzelte Ergebnisse aus Befragungen zu Influenzaimpfquoten für Patienten in bPAEinrichtungen vor.

Fragestellung. Ziel der Studie war es, auf Basis ambulanter Abrechnungsdaten erstmals einen bundesweiten Überblick über die Influenzaimpfquoten bei in bPAEinrichtungen versorgten Patienten zu geben. Material und Methoden. Es erfolgte die deskriptive Auswertung der ambulanten vertragsärztlichen Abrechnungsdaten für 6 Studienkohorten in den Jahren 2010 bis 2016.

Ergebnisse. Die Impfquote der Studienkohorten variiert über die Saisons hinweg zwischen $56,6 \%$ und $60,3 \%$. Über den Gesamtzeitraum wurde ein Durchschnitt von $58,5 \%$ erreicht. Es zeigten sich deutliche regionale Unterschiede in den Influenzaimpfquoten von bis zu 20 Prozentpunkten (PP) sowie wenn zuvor ein Hausarzt konsultiert wurde (bis zu 35 PP). Diskussion. Die Ergebnisse geben erste Einblicke zu Impfquoten in bPA-Einrichtungen und enthalten Hinweise auf Zusammenhänge zwischen strukturellen Versorgungsunterschieden und der Inanspruchnahme von Influenzaimpfungen. Die Ergebnisse können dazu beitragen, Gruppen mit besonders niedrigen Influenzaimpfquoten zu identifizieren. Sie ergänzen die Datenbasis für gesundheitspolitische Maßnahmen zur Erhöhung der Impfquoten. Für die untersuchte Gruppe wurden die Empfehlungen der EK zu einer Influenzaimpfquote von $75 \%$ bisher nicht erreicht.

\section{Schlüsselwörter}

Routinedaten - Influenzaimpfquoten .

Versorgung in institutionellen Einrichtungen . Ältere · Ambulante Versorgung

\section{Influenza vaccination rates of patients with statutory ambulatory medical care in protective facilities and in nursing and special care homes}

\section{Abstract}

Background. Vaccination is one of the most important and effective preventive measures against seasonal influenza. The German Standing Committee on Vaccination (STIKO) recommends influenza vaccination for specific groups of patients, including those in protective facilities and nursing or special care homes ("bPA-facilities"). The defined aim of the European Commission (EC) is vaccination coverage of $75 \%$ or higher among the elderly. In Germany, vaccination rates in bPA-facilities are currently based on surveys in limited samples of patients.

Objectives. The aim of the present study is to gain better insight into nationwide influenza vaccination rates. Specifically, we aim to identify influenza vaccination coverage of patients with ambulatory medical care in bPAfacilities.

Material and methods. The present analysis is a descriptive analysis of Germany's billing data of all ambulatory statutory health insurance physicians (ASHIP) for six study cohorts from 2010 to 2016.

Results. On average, $58.5 \%$ of patients in bPAfacilities received an influenza vaccination between 2010 (60.3\%) and 2016 (56.6\%), with strong differences between different ASHIP billing areas (up to 20 percentage points(pp)) and patients who consulted a general practitioner (up to $35 \mathrm{pp}$ ).
Conclusions. These results provide initial insights into vaccination coverage in bPAfacilities and show associations between the structural differences in care setting and the use of influenza vaccines. Furthermore, they can help to identify groups with low influenza vaccination rates and complement existing data thereby assisting health policy in increasing vaccination coverage. The present study group has not yet reached the EC goal of $75 \%$ influenza vaccination coverage.

Keywords

Routine data · Influenza vaccination coverage Patient in nursing homes · Elderly · Ambulatory medical care und $2015(N=866.300)$ angegeben. Die Studienkohorten der Jahre 2011/2012, 2013/2014 und 2015/2016 entsprechen durchschnittlich $56 \%$ der in der öffentlichen Pflegestatistik erfassten verfügbaren vollstationären Plätze in Pflegeheimen. Das Durchschnittsalter betrug in den jeweiligen Impfsaisons 84 Jahre, mit einer Standardabweichung von 8 Jahren (Median: 85 Jahre). Die Hälfte der Patienten hatte ein Alter zwischen 79 und 90 Jahren in den betrachteten Impfsaisons.

\section{Impfquoten}

Die bundesweite Impfquote der ab 60jährigen, in bPA-Einrichtungen vertragsärztlich versorgten Patienten lag in der ersten untersuchten Impfsaison 2010/2011 bei 60,3\%. Über die Jahre zeigte sich ein Abfall der Impfquoten auf 56,6\% (Impfsaison 2015/2016). Durchschnittlich 59,0\% der in bPA-Einrichtungen vertragsärztlich versorgten Patienten mit chronischen Erkrankungen wurden gegen die Influenza in den Impfsaisons von 2010/2011 bis 2015/2016 geimpft. - Tab. 1 stellt die Anzahl der in bPAEinrichtungen versorgten Patienten und den prozentualen Anteil der gegen Influenza geimpften Patienten differenziert nach Impfsaison, Alter, Geschlecht und weiteren Merkmalen dar.

Die relative Häufigkeit der geimpften Frauen in bPA-Einrichtungen betrug durchschnittlich $59,1 \%$ und die der geimpften Männer $56,8 \%$ in den 


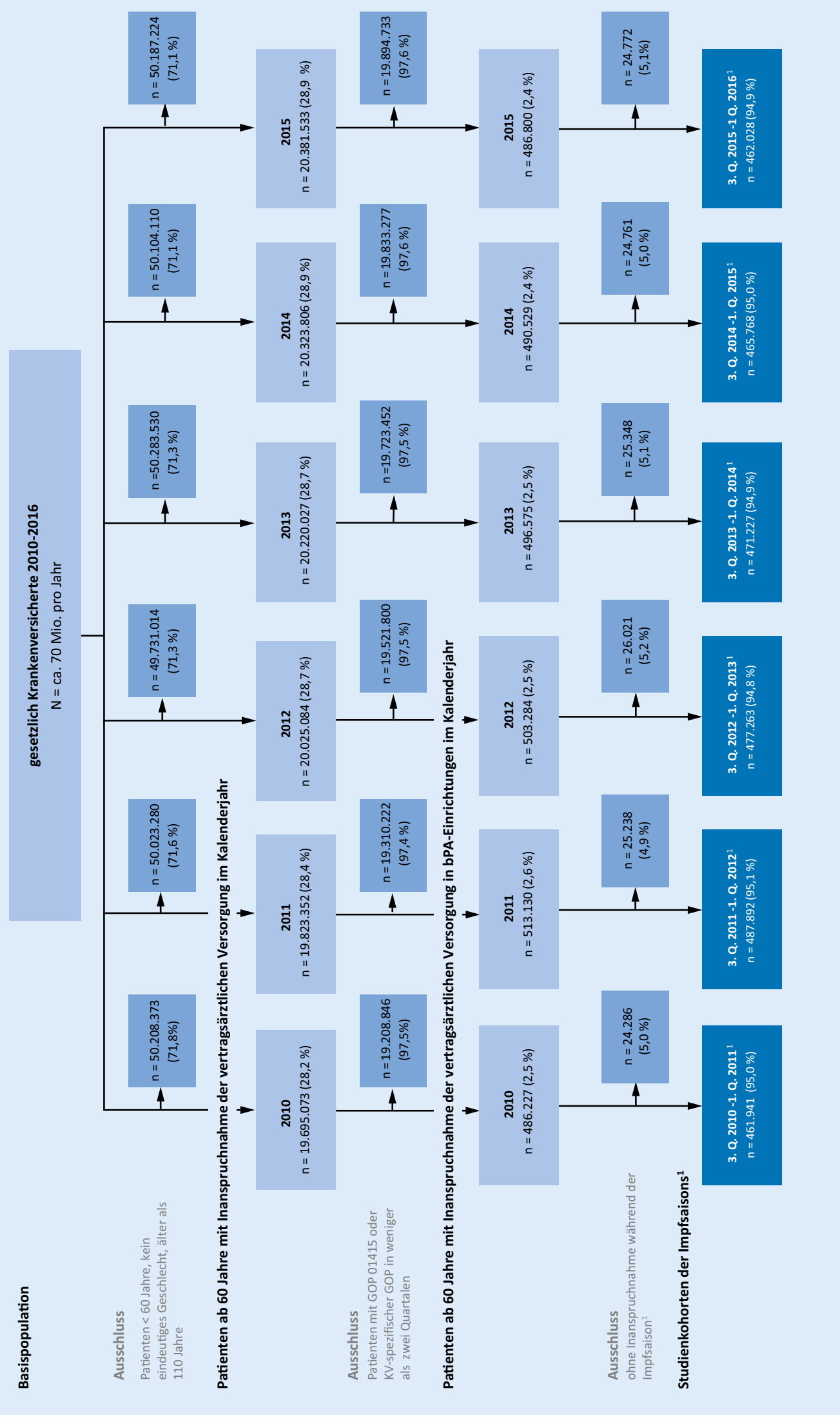

Abb. $1<$ Bildung der einzelnen Studienkohorten zu den Impfsaisons 2010/2011 bis 2015/2016. Flussdiagramm. ${ }^{1}$ Die Impfsaison umfasst den Zeitraum 3. Quartal des Jahres bis einschließlich des 1. Quartals des Folgejahres, $b P A$ beschützende Einrichtungen, Alten- und Pflegeheime, Q Quartal, KV Kassenärztliche Vereinigung, Mio. Millionen, GOP Gebührenordnungsposition, ca. circa 
Tab. 1 Anzahl in bPA-Einrichtungen versorgter Patienten und prozentualer Anteil der gegen Influenza geimpften Patienten differenziert nach Impfsaison, Alter, Geschlecht und weiteren Merkmalen. bPA: beschützenden Einrichtungen, Alten- und Pflegeheime

\begin{tabular}{|c|c|c|c|c|c|c|c|c|c|c|c|c|}
\hline \multirow[t]{2}{*}{ Impfsaison } & \multicolumn{2}{|c|}{$2010 / 2011$} & \multicolumn{2}{|c|}{$2011 / 2012$} & \multicolumn{2}{|c|}{$2012 / 2013$} & \multicolumn{2}{|c|}{$2013 / 2014$} & \multicolumn{2}{|c|}{$2014 / 2015$} & \multicolumn{2}{|c|}{ 2015/2016 } \\
\hline & \multicolumn{12}{|c|}{$\boldsymbol{N}$ (\% Anteil gegen Influenza geimpfter Patienten) } \\
\hline \multicolumn{13}{|c|}{ Patienten in bPA-Einrichtungen } \\
\hline Studienkohorte & 461.941 & $(60,3)$ & 487.892 & $(60,6)$ & 477.263 & $(57,0)$ & 471.227 & $(58,6)$ & 465.768 & $(57,6)$ & 462.028 & $(56,6)$ \\
\hline \multicolumn{13}{|l|}{ Geschlecht } \\
\hline Männer & 117.417 & $(58,7)$ & 126.512 & $(58,6)$ & 126.574 & $(55,4)$ & 126.868 & $(57,0)$ & 127.594 & $(56,0)$ & 128.464 & $(54,9)$ \\
\hline Frauen & 344.524 & $(60,9)$ & 361.380 & $(61,3)$ & 350.689 & $(57,6)$ & 344.359 & $(59,2)$ & 338.174 & $(58,3)$ & 333.564 & $(57,2)$ \\
\hline \multicolumn{13}{|l|}{ Altersgruppen } \\
\hline $60-69$ & 31.402 & $(57,8)$ & 32.309 & $(57,9)$ & 31.786 & $(55,2)$ & 31.318 & $(56,3)$ & 30.795 & $(54,7)$ & 31.789 & $(53,2)$ \\
\hline $70-79$ & 94.479 & $(59,4)$ & 99.845 & $(59,4)$ & 98.688 & $(56,1)$ & 98.402 & $(57,4)$ & 95.890 & $(56,2)$ & 92.219 & $(55,1)$ \\
\hline $80-89$ & 226.908 & $(61,1)$ & 234.039 & $(61,4)$ & 222.825 & $(57,8)$ & 215.778 & $(59,4)$ & 211.934 & $(58,7)$ & 209.374 & $(57,4)$ \\
\hline$\geq 90$ & 109.152 & $(60,2)$ & 121.699 & $(60,6)$ & 123.964 & $(56,8)$ & 125.729 & $(58,8)$ & 127.149 & $(57,7)$ & 128.646 & $(57,2)$ \\
\hline \multicolumn{13}{|l|}{ Chroniker } \\
\hline Chroniker & 416.777 & $(61,0)$ & 443.613 & $(61,1)$ & 435.030 & $(57,6)$ & 433.485 & $(59,1)$ & 430.603 & $(58,1)$ & 428.780 & $(57,1)$ \\
\hline \multicolumn{13}{|l|}{ Siedlungsstruktur } \\
\hline Kernstadt & 114.664 & $(57,1)$ & 116.648 & $(57,0)$ & 113.301 & $(54,5)$ & 112.889 & $(55,7)$ & 112.289 & $(54,2)$ & 110.702 & $(53,2)$ \\
\hline Verdichtetes Umland & 262.969 & $(60,1)$ & 278.608 & $(60,3)$ & 273.018 & $(56,5)$ & 269.335 & $(58,0)$ & 264.869 & $(57,2)$ & 262.554 & $(56,0)$ \\
\hline Ländliches Umland & 84.308 & $(65,6)$ & 92.636 & $(66,0)$ & 90.944 & $(61,7)$ & 89.003 & $(64,0)$ & 88.610 & $(63,5)$ & 88.772 & $(62,5)$ \\
\hline \multicolumn{13}{|l|}{ Ost/West } \\
\hline West & 385.543 & $(58,7)$ & 411.468 & $(59,0)$ & 401.620 & $(55,0)$ & 393.166 & $(56,7)$ & 387.427 & $(55,6)$ & 382.822 & $(54,4)$ \\
\hline Ost & 76.398 & $(68,7)$ & 76.424 & $(69,1)$ & 75.643 & $(67,5)$ & 78.061 & $(68,0)$ & 78.341 & $(67,9)$ & 79.206 & $(67,2)$ \\
\hline
\end{tabular}

untersuchten Impfsaisons. Die relative Impfquote in der ersten Impfsaison (2010/2011) und in der letzten Impfsaison (2015/2016) lag bei Männern bei $58,7 \%$ bzw. bei $54,9 \%$. Die Impfquoten fielen somit deutlich niedriger als bei Frauen mit $60,9 \%$ sowie $57,2 \%$ aus. Die - Abb. 2 stellt die absoluten Zahlen und relativen Häufigkeiten differenziert nach Geschlecht pro Impfsaison dar.

Die Betrachtung der Altersverteilung der Influenzaimpfquoten in den Impfsaisons ergab, dass die höchsten Impfquoten in den Altersgruppen ab 80 Jahren vorlagen. Die relativen Influenzaimpfquoten zwischen der ersten Impfsaison (2010/2011) und der letzten Impfsaison (2015/2016) zeigten eine deutliche Abnahme der Impfungen in den Altersgruppen um durchschnittlich 4 Prozentpunkte (•Tab. 1).

- Abb. 3 verdeutlicht, dass bei Inanspruchnahme von hausärztlicher Versorgung die Impfquoten in allen Impfsaisons höher lagen als ohne Inanspruchnahme. Im Durchschnitt wurden 58,7 \% der in bPA-Einrichtungen vertragsärztlich versorgten Patienten, die einen Hausarzt konsultiert hatten, in den Impfsai- sons von 2010/2011 bis 2015/2016 gegen Influenza geimpft. Einer von hundert Patienten nahm durchschnittlich die hausärztliche Versorgung in den Impfsaisons nicht in Anspruch. Bei den Patienten ohne hausärztliche Versorgung sank die Impfquote innerhalb der Impfsaisons auf durchschnittlich $24,8 \%$.

Stratifiziert nach der Siedlungsstruktur lagen die Impfquoten durchschnittlich bei 55,3\% in Kernstädten, bei 58,0 \% im verdichteten Umland und bei $63,9 \%$ imländlichen Umland [24].• Abb. 4 stellt die Influenzaimpfquoten in den Kassenärztlichen Vereinigungen (KVen) dar. Die niedrigsten Influenzaimpfquoten waren in den untersuchten Impfsaisons durchgängig in Hamburg (2010/2011: 54,0\%; 2015/2016: 48,0\%), Nordrhein (2010/2011: 55,1\%; 2015/2016: 50,4\%) und Bremen (2010/2011: 55,2\%; 2015/ 2016: 49,8\%) zu finden.

Die höchsten Influenzaimpfquoten fanden sich insgesamt in den neuen Bundesländern. Der stärkste Rückgang der Impfquoten zwischen den Impfsaisons 2010/2011 und 2015/2016 von durchschnittlich 4,6 Prozentpunkten ist in den alten Bundesländern zu verzeichnen. In den neuen Bundesländern sanken die Impfquoten um durchschnittlich 2,4 Prozentpunkte. Über die Untersuchungszeiträume hinweg waren die Impfquoten in den betrachteten Altersgruppen in den neuen Bundesländern durchgängig höher als in den alten Bundesländern (•Tab. 1).

\section{Diskussion}

Die vorliegende Studie liefert erste Einblicke zur Umsetzung der Empfehlung von Influenzaimpfungen der STIKO von in deutschen bPA-Einrichtungen vertragsärztlich versorgten Patienten. Die hier beschriebenen Studienkohorten stellen Subgruppen der Gesamtheit aller Patienten in bPA-Einrichtungen dar. Bisher lagen für Deutschland lediglich Ergebnisse aus Einzelerhebungen in bPA-Einrichtungen vor. Trotz der Einschränkungen auf die beschriebenen Subgruppen bietet sich aufgrund der Größe der Beobachtungsgruppe und der hier angewandten Methode die Gelegenheit, einen relevanten Eindruck über das Impfgeschehen in bPA-Einrichtungen zu erlangen. Die Influenzaimpfempfehlun- 


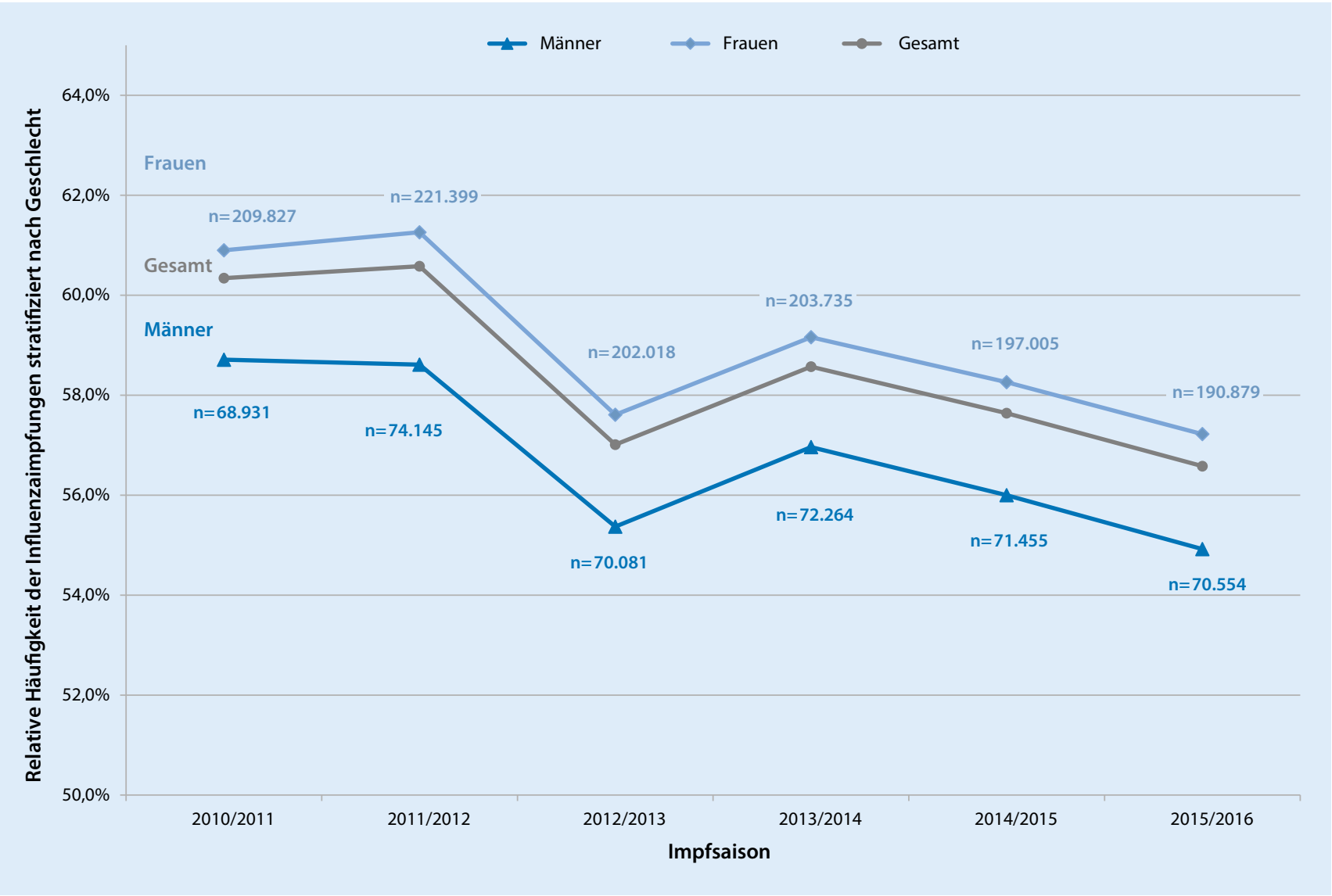

Abb. 2 - Impfquoten für Impfungen gegen saisonale Influenza von in bPA-Einrichtungen versorgten Patienten nach Geschlecht und Impfsaison. $b P A$ beschützende Einrichtungen, Alten- und Pflegeheime

gen wurden durchschnittlich mit einer Quote von 58,5\% über alle beobachteten Impfsaisons umgesetzt. Im Rahmen des bundesweiten Gesundheitsmonitorings (GEDA) des Robert Koch-Instituts (RKI) konnten ähnliche Impfquoten von $54,3 \%(2010 / 2011)$ bzw. $52,6 \%$ (2011/2012) der $\geq 60$-jährigen Personen ermittelt werden $[26,27]$. Riens et al. schätzen anhand von vertragsärztlichen Abrechnungsdaten Impfquoten von $47 \%$ (2009/2010) bzw. 37,8\% (2013/2014) der $\geq 60$-jährigen Patienten [11]. Der Unterschied der Impfquoten lässt sich durch die methodische Herangehensweise der Studien erklären. Der prozentuale Anteil gegen Influenza geimpfter in bPAEinrichtungen versorgter Patienten sank in den letzten 6 Jahren kontinuierlich (2010/2011: 60,3\%; 2015/2016: 56,6\%). Erklären lässt sich die Anzahl nichtgeimpfter Patienten in den Studienkohorten zum Teil durch die Freiwilligkeit der Impfung, wobei die Entscheidung im Rahmen einer gemeinsamen Entschei- dungsfindung erfolgen soll, sowie durch möglicherweise vorliegende Kontraindikationen [28, 29]. Umfrageergebnisse der Bundeszentrale für gesundheitliche Aufklärung (BzGA) zeigen, dass nahezu zwei Drittel der Befragten ab 60 Jahren die Grippeimpfung für sich persönlich als „(besonders) wichtig“ einschätzen (63\%). Die große Mehrzahl der Patienten in bPA-Einrichtungen weist geriatrische Krankheitsbilder auf. Die Patienten verbringen oft ihre letzte Lebensphase in den Einrichtungen, dies kann vermutlich die Einschätzung der Wichtigkeit der saisonalen Grippeimpfung beeinflussen. Ein Grund für die Nichtinanspruchnahme der Influenzaimpfungen bei den über 60-Jährigen ( $51 \%$ ) und bei Chronikern (48\%) war der Zweifel an der Wirksamkeit der Impfung. Die Einschätzung, dass Grippe keine besonders schwere Krankheit sei, lag in beiden Gruppen bei $35 \%$ [30]. Ursachen für die in der vorliegenden Arbeit gefundenen rückläufigen Impfquoten können allerdings im Rahmen dieser Arbeit nicht benannt werden.

Unsere Analysen weisen darauf hin, dass das Impfverhalten stark durch die Inanspruchnahme der hausärztlichen Versorgung (58,7\% mit vs. $24,8 \%$ ohne hausärztliche Versorgung) geprägt wird. Hausärzte spielen eine primäre Rolle hinsichtlich der Impfmotivation [14, 16]. Patienten mit chronischen Erkrankungen sowie in bPA-Einrichtungen lebende Patienten sollten über den Nutzen der Impfung im Vergleich zum Risiko der Krankheit aufgeklärt werden, um die Entscheidungsfindung $\mathrm{zu}$ unterstützen [30, 31].

Deutliche regionale Unterschiede in den Influenzaimpfquoten zeigen sich konsistent in allen Studienkohorten. In den neuen Bundesländern waren die Impfquoten über die Untersuchungszeiträume durchgängig höher als in den alten Bundesländern. Die Ost-WestUnterschiede der Influenzaimpfquoten könnten auf historische Gegebenheiten 


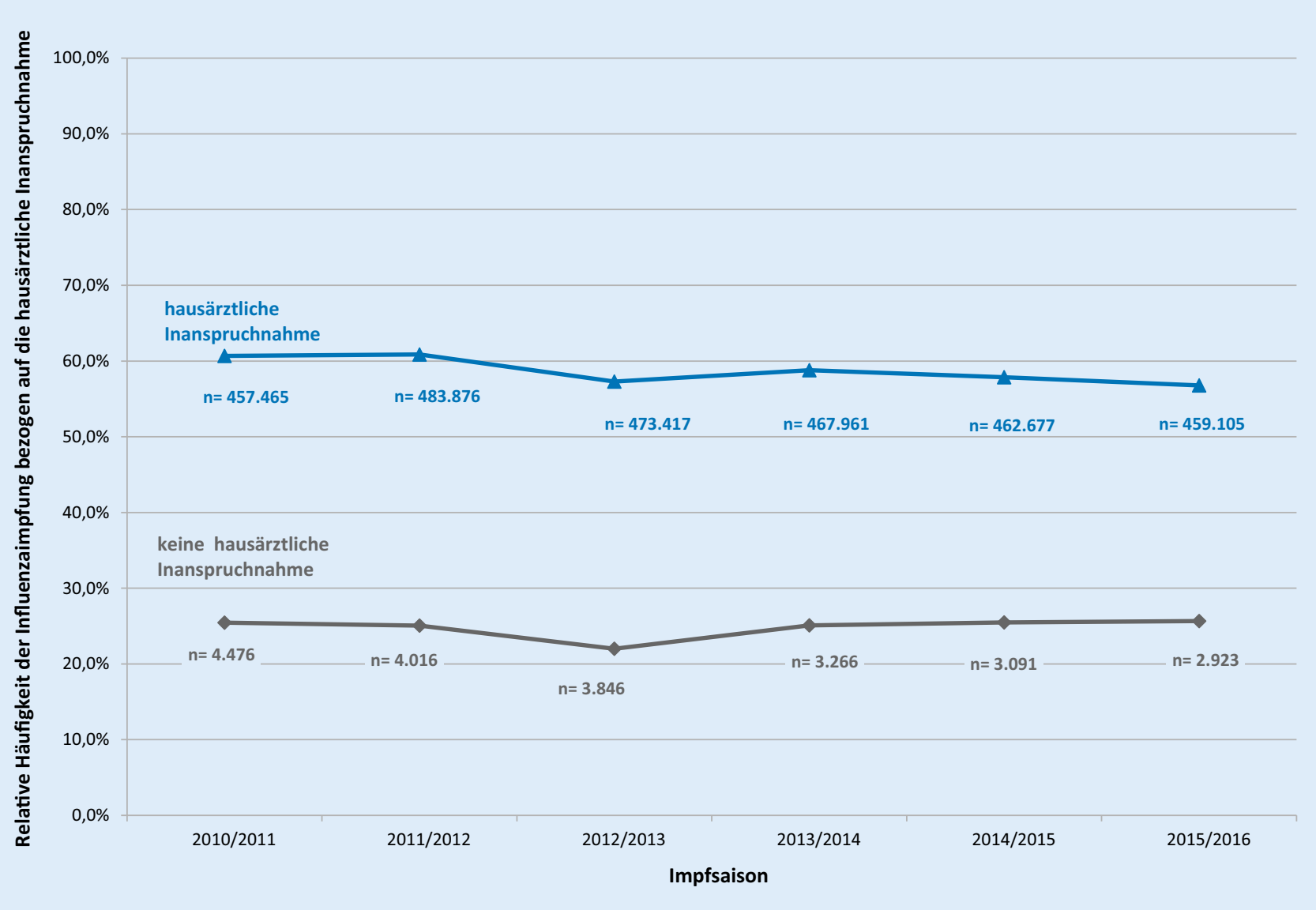

Abb. 3 ॥ Impfquoten für Impfungen gegen saisonale Influenza von in bPA-Einrichtungen versorgten Patienten nach hausärztlicher Inanspruchnahme. $P P A$ beschützende Einrichtungen, Alten- und Pflegeheime

(Impfpflicht vs. Impfempfehlung) zurückgeführt werden, welche sich in der Inanspruchnahme der Influenzaimpfung widerspiegeln. Für die Influenzaimpfquoten in den KVen Bayerns und BadenWürttemberg liegt wahrscheinlich eine Unterschätzung vor. Die Daten der im Rahmen der sogenannten hausarztzentrierten Versorgung $(\mathrm{HzV})$ behandelten Patienten sind nicht Bestandteil der verwendeten Datengrundlage. Im Rahmen der KV-Impfsurveillance zeigte sich bei den über 60-Jährigen bereits ein rückläufiger Trend. Der abnehmende Trend der Inanspruchnahme von Influenzaimpfungen zeigt sich ebenso bei in bPAEinrichtungen versorgten Patienten [11, 32, 33].

Von den europäischen Ländern wurde der Influenzaimpfschutz in den höheren Altersgruppen (entsprechend den Empfehlungen des jeweiligen Mitgliedstaates z. B. $\geq 59, \geq 60$ oder $\geq 65$ Jahre) für die Saison 2012/2013 an das European
Centre for Disease Prevention and Control (ECDC) gemeldet. Die Impfquoten in den Ländern variierten von 1-77,4\% (Median:44,7\%). Diehöchsten Impfquoten wurden von den Niederlanden (77\%) und England (74\%) gemeldet [34]. In den USA belief sich die Impfquote der Saison 2015/2016 bei den über 65-Jährigen auf $63,4 \%$, was im Vergleich zu der Saison 2014/2015 einen Rückgang um 3,3\% bedeutet [35]. Internationale Studien zeigen Impfquoten von ca. $80 \%$ bis über $90 \%$ für Patienten in bPA-Einrichtungen - Kanada (92\%; [20]), Frankreich (80,8-91\%; $[36,37])$, USA (76,2\%; [38]).

Im internationalen Vergleich zeigen sich in dieser Untersuchung für Deutschland niedrigere Impfquoten in den Studienkohorten der in bPA-Einrichtungen vertragsärztlich versorgten Patienten, welche auch im Zeitverlauf rückläufig sind. In anderen europäischen Ländern zeichnet sich ein ähnliches Bild für ältere Patienten ab [39]. Unterschied- liche nationale Studien beobachteten seit der pandemischen Influenzasaison 2009/2010 eine Abnahme der Impfquoten. Die kontroversen Diskussionen über Sicherheit und Wirksamkeit einer Impfung könnten die Impfbereitschaft in der Bevölkerung negativ beeinflusst haben [30].

Die Auswertung der Studienkohorten gibt erste Hinweise auf die Abdeckung der Influenzaimpfungen. Die gewählte Methodik lässt jedoch keine vollständigen Aussagen bezogen auf alle Patienten in bPA-Einrichtungen zu. Vor dem Hintergrund, dass jedem Patienten in bPAEinrichtungen eine Influenzaimpfung empfohlen wird, deren Nutzen die Risiken deutlich überwiegt, sofern keine Kontraindikation vorliegt, lassen die anhand unserer Beobachtung ermittelten Impfquoten einen Nachholbedarf vermuten, bezogen auf die durch die $\mathrm{Eu}$ ropäische Kommission geforderte $75 \%$ Abdeckung. 


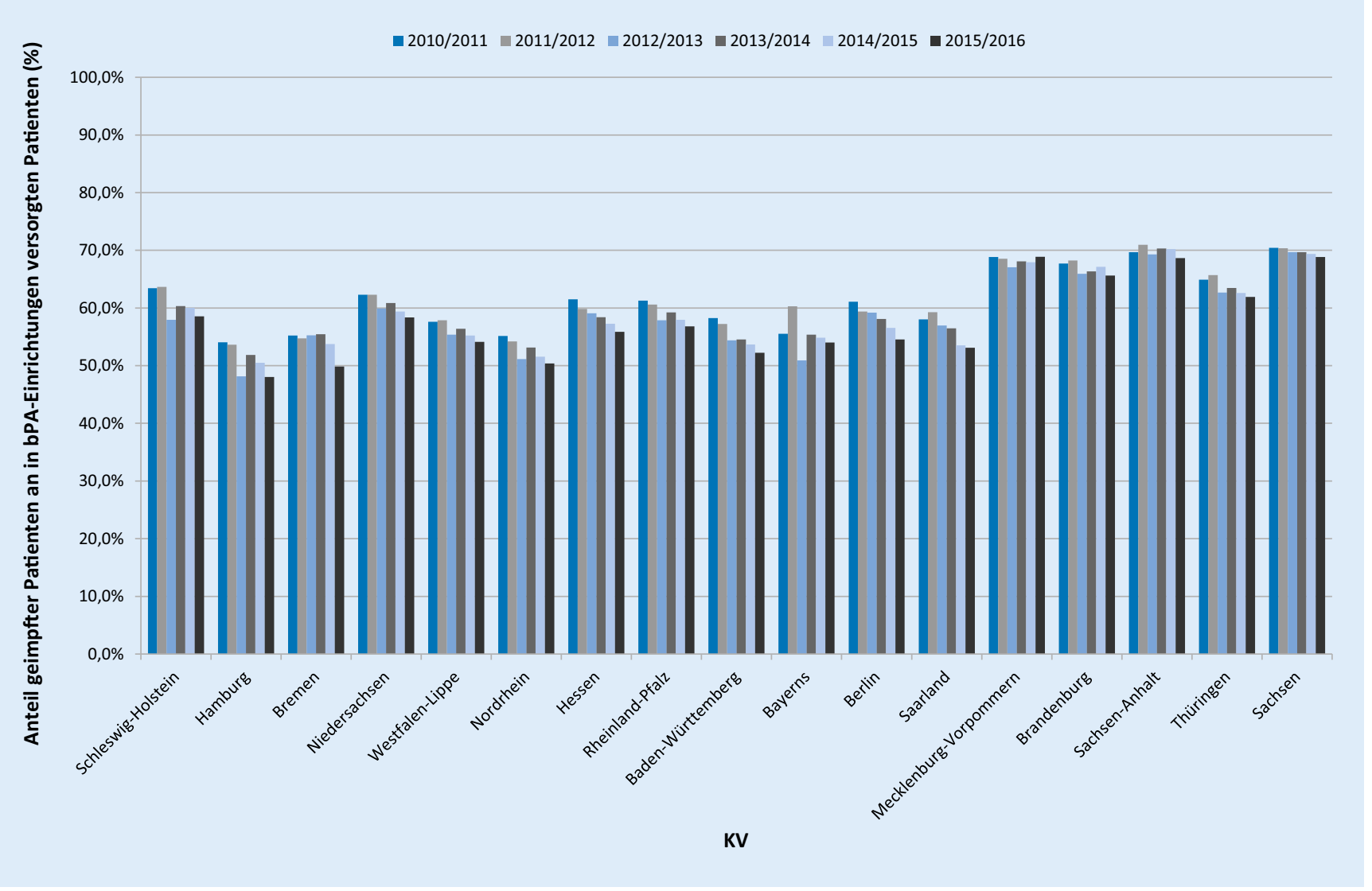

Abb. $4 \Delta$ Impfquoten für Impfungen gegen saisonale Influenza von in bPA-Einrichtungen versorgten Patienten stratifiziert nach KV-Regionen. $b P A$ beschützende Einrichtungen, Alten- und Pflegeheime, KV Kassenärztliche Vereinigung

\section{Stärken und Limitationen}

Die verwendeten Routinedaten der vorliegenden Studie bilden erstmals die krankenkassen- und regionalübergreifenden Influenzaimpfquoten von vertragsärztlich versorgten Patienten in bPA-Einrichtungen ab. Auch wenn es sich hier lediglich um eine Subgruppe aller Patienten in bPA-Einrichtungen handelt, können erste Eindrücke zum Impfgeschehen auf dieser Basis gewonnen werden. Die Arbeit leistet einen Beitrag, die Umsetzung von Influenzaimpfempfehlungen in bPA-Einrichtungen auch im zeitlichen Verlauf abzuschätzen. Bisher lagen keine Studien von Influenzaimpfquoten von Patienten in Altenund Pflegeheimen in diesem Umfang und über einen solchen Zeitverlauf vor.

Die Analysen beziehen sich auf einen Zeitraum von 6 Jahren und erlauben die Beurteilung der Entwicklung des Impfgeschehens für die Subgruppe der in bPAEinrichtungen vertragsärztlich versorg- ten Patienten. Eine Einschränkung der vorliegenden Studie liegt in der grundsätzlichen Begrenzung der Aussagefähigkeit von Routinedaten, welche nicht primär für Forschungszwecke erfasst werden und somit mehrere Schwächen aufweisen. So können Influenzaimpfungen möglicherweise im stationären Umfeld aufgrund eines Krankenhaus- und/ oder Rehabilitationsaufenthaltes erfolgt und deshalb nicht in die Daten eingeflossen sein. Zudem sind die Ergebnisse abhängig von der Definition und Auswahl der untersuchten Grundgesamtheit sowie der Operationalisierung der $\mathrm{Zu}$ ordnung von in bPA-Einrichtungen versorgten Patienten. Mit der gewählten Operationalisierung wurde eine inhaltlich medizinisch abgeleitete, transparente und über den Untersuchungszeitraum konstante Kohortenbildung angestrebt, um eine möglichst valide Schätzung der Grundgesamtheit von in bPA-Einrichtungen versorgten Patienten, auch im Zeitverlauf, zu erreichen. Durch die
Abrechnung der Besuchspauschale über mindestens 2 Quartale des Aufgreifjahres hinweg wird sichergestellt, dass beispielsweise Patienten in der Kurzzeitpflege nicht eingeschlossen sind. Eine weitere Absicherung, dass die Patienten mindestens eine ambulante Leistung in der Impfsaison erhalten haben, stellte sicher, dass diese sich während der Impfsaison in der bPA-Einrichtung befanden.

Verglichen mit den öffentlichen Statistiken zur Anzahl der vollstationären Pflegeheimplätze ergibt sich für die Jahre 2011, 2013 und 2015 eine durchschnittliche Abdeckung von ca. 56\%. Zum Teil lässt sich die Differenz zur Anzahl der vollstationären Pflegeheimplätze von $44 \%$ dadurch erklären, dass durch die gewählte Operationalisierung zur Zuordnung von Patienten in bPAEinrichtungen solche Patienten, die in bPA-Einrichtungen leben, deren Inanspruchnahme der vertragsärztlichen Versorgung aber rein in Praxen erfolgt, nicht berücksichtig werden. Zu beachten 
ist zudem, dass Altenheime oder andere Wohnformen in die öffentlichen Statistiken der vollstationären Pflegeheimplätze nicht eingehen, sodass der Anteil der Studienkohorte an der wahren Anzahl der in Alten- und Pflegeeinrichtungen lebenden Menschen noch deutlich geringer sein wird. Zudem werden privat krankenversicherte Patienten sowie Patienten in regionalen Selektivverträgen und/oder Verträgen der $\mathrm{HzV}$ ebenfalls nicht in den vorliegenden Routinedaten erfasst [25].

\section{Fazit}

Die vorgestellten Impfquoten aus der Routinedatenerhebung geben die Möglichkeit, erste Erkenntnisse zu in bPAEinrichtungen vertragsärztlich versorgten Patienten zu erhalten. Die Ergebnisse zeigen eine Abnahme der Impfquoten von der Impfsaison 2010/2011 bis zur Impfsaison 2015/2016 um 3,7 Prozentpunkte. Weiterhin zeigten sich Hinweise auf einen Zusammenhang hinsichtlich der strukturellen Versorgungsunterschiede und der Inanspruchnahme von Influenzaimpfungen. Für die untersuchte Gruppe wurden die Empfehlungen der Europäischen Kommission zu einer Influenzaimpfquote von $75 \%$ bisher nicht erreicht.

Die Ergebnisse unterstreichen die Notwendigkeit von weiteren gesundheitspolitischen Maßnahmen und einer verstärkten Verbreitung von Gesundheitsinformationen zur Erhöhung der Impfbereitschaft und verbesserten Umsetzung von Impfungen in bPA-Einrichtungen. Dies ist erforderlich, um Morbidität, Mortalität und sensitive Krankenhauseinweisungen aufgrund vermeidbarer Influenzaerkrankungen zukünftig weiter zu verringern.

\section{Korrespondenzadresse}

\section{Dipl.-Wirtschaftsinformatikerin (FH) Diana Kurch-Bek, MPH}

Innovation, strategische Analyse und ITBeratung, Kassenärztliche Bundesvereinigung Herbert-Lewin-Platz 2, 10623 Berlin,

Deutschland

dkurch-bek@kbv.de

\section{Einhaltung ethischer Richtlinien}

Interessenkonflikt. D. Kurch-Bek, C. Gallowitz, B. Tenckhoff und L. Sundmacher geben an, dass kein Interessenkonflikt besteht.

Dieser Beitrag beinhaltet keine von den Autoren durchgeführten Studien an Menschen oder Tieren. Dieser Beitrag berücksichtigt die Gute Praxis Sekundärdatenanalyse (GPS).

Open Access Dieser Artikel wird unter der Creative Commons Namensnennung 4.0 International Lizenz (http://creativecommons.org/licenses/by/4.0/deed. de) veröffentlicht, welche die Nutzung, Vervielfältigung, Bearbeitung, Verbreitung und Wiedergabe in jeglichem Medium und Format erlaubt, sofern Sie den/die ursprünglichen Autor(en) und die Quelle ordnungsgemäßnennen, einen Linkzur Creative Commons Lizenz beifügen und angeben, ob Änderungen vorgenommen wurden.

\section{Literatur}

1. Gaillat J, Chidiac C, Fagnani F et al (2009) Morbidity and mortality associated with influenza exposure in long-term care facilities for dependent elderly people. Eur J Clin Microbiol Infect Dis 28(9):1077-1086. https://doi.org/10.1007/ s10096-009-0751-3

2. Utsumi M, Makimoto K, Quroshi N, Ashida N (2010) Types of infectious outbreaks and their impact in elderly care facilities: a review of the literature. Age Ageing 39(3):299-305. https://doi.org/10.1093/ ageing/afq029

3. Win MK, Chow A, Chen M, Lau YF, OOi EE, Leo YS (2010) Infuenza B outbreak among influenzavaccinated welfare home residents in Singapore

4. Tengs TO, Adams ME, Pliskin JS et al (1995) Fivehundred life-saving interventions and their costeffectiveness. RiskAnal 15(3):369-390. https://doi. org/10.1111/j.1539-6924.1995.tb00330.x

5. Peasah SK, Azziz-Baumgartner E, Breese J, Meltzer Ml, Widdowson M-A (2013) Influenza cost and cost-effectiveness studies globally - a review. Vaccine 31(46):5339-5348. https://doi.org/10. 1016/j.vaccine.2013.09.013

6. Kommission der Europäischen Gemeinschaften (2009) Empfehlung des Rates zur Impfung gegen die saisonale Grippe. http://ec. europa.eu/health/ph_threats/com/Influenza/ docs/seasonflu_rec2009_de.pdf.Zugegriffen: 30 . Jan. 2017

7. Kassenärztliche Bundesvereinigung AQUIK-Indikatorenset (2009) http://www.kbv.de/html/aquik. php.Zugegriffen:20.Dez. 2017

8. Robert Koch-Institut (2016) Empfehlungen der Ständigen Impfkommission (STIKO) am RKI. Stand August 2016

9. OECD Data for measuring health care quality and outcomes. Health carequalityindicators-primary care. http://www.oecd.org/els/health-systems/ hcqi-primary-care.htm. Zugegriffen: 20. Dez. 2017

10. NHS Quality and Outcomes Framework (QOF) http://content.digital.nhs.uk/qof. Zugegriffen: 20. Dez. 2017

11. Riens B, Mangiapane $S$, Erhart $M$, von Stillfried D (2012) Analyse regionaler Unterschiede der Influenza-Impfraten in der Impfsaison 2007/2008. http://www.versorgungsatlas.de/fileadmin/ziva_ docs/ID1u2_Dok1_Bericht_Langversion_.pdf. Zugegriffen: 13. Febr. 2017
12. Bätzing-Feigenbaum J, Schulz $M$, Schulz $M$, Gisbert M (2015) Entwicklung der saisonalen Influenzastandardimpfraten im kassenärztlichen Versorgungssektor in Deutschland seit der Pandemie 2009-Eine Trendanalyse auf regionaler Ebene für den Zeitraum 2009/2010 bis 2013/2014

13. Bödeker B, Wichmann O, Mertens B, Seefeld L, Pott E (2014) Umsetzung der Influenzaimpfempfehlung in Alten- und Pflegeheimen. Ergebnisse einer Erhebung im Rahmen der nationalen InfluenzaImpfaufklarungskampagne (Implementation of the influenza vaccination recommendation in nursing homes in Germany : results of a survey as part of the national influenza immunization campaign). Bundesgesundheitsblatt Gesundheitsforschung Gesundheitsschutz 57(11):1315-1321. https:// doi.org/10.1007/s00103-014-2044-y

14. Thode N, Bergmann E, Kamtsiuris $P$, Kurth B-M (2005) Einflussfaktoren auf die ambulante Inanspruchnahme in Deutschland (Predictors for ambulatory medical care utilization in Germany). Bundesgesundheitsblatt Gesundheitsforschung Gesundheitsschutz 48(3):296-306. https://doi. org/10.1007/s00103-004-1004-3

15. Bohmer MM, Walter D, Krause G, Muters S, Gosswald A, Wichmann $O$ (2011) Determinants of tetanus and seasonal influenza vaccine uptake in adults living in Germany. Hum Vaccin 7(12):1317-1325. https://doi.org/10.4161/hv.7. 12.18130

16. Gulich M, Eberhardt S, Blankenhorn S, Zeitler H-P (2006) Motivation zur Grippeimpfung bei hausärztlichen Patienten in der Saison 2005/2006. Z Allg Med 82(8):334-337. https://doi.org/10. 1055/s-2006-942084

17. Nagata JM, Hernández-RamosI, KurupAS, Albrecht D, Vivas-Torrealba C, Franco-Paredes C(2013) Social determinants of health and seasonal influenza vaccination in adults $\geq 65$ years: a systematic review of qualitative and quantitative data. BMC Public Health 13(1):388. https://doi.org/10.1186/ 1471-2458-13-388

18. Wiese-Posselt M, Leitmeyer $\mathrm{K}$, Hamouda $\mathrm{O}$ et al (2006) Influenza vaccination coverage in adults belonging to defined target groups, Germany, 2003/2004. Vaccine 24(14):2560-2566. https:// doi.org/10.1016/j.vaccine.2005.12.020

19. Rainwater-Lovett K, Chun K, Lessler J (2014) Influenza outbreak control practices and the effectiveness of interventions in long-term care facilities: a systematic review. Influenza Other Respir Viruses 8(1):74-82. https://doi.org/10. 1111/irv. 12203

20. Mahmud SM, Thompson LH, Nowicki DL, Plourde PJ (2013) Outbreaks of influenza-like illness in longterm care facilities in Winnipeg, Canada. Influenza Other Respir Viruses 7(6):1055-1061. https://doi. org/10.1111/irv.12052

21. Deutsches Institut für medizinische Dokumentation und Information (2018) Internationale statistische Klassifikation der Krankheiten und verwandter Gesundheitsprobleme, 10. Revision, German Modification (ICD-10-GM). http://www. dimdi.de/static/de/klassi/icd-10-gm/index.htm. Zugegriffen: 17.Sept. 2018

22. Kassenärztliche Bundesvereinigung (2018) Einheitlicher Bewertungsmaßstab (EBM). http:// www.kbv.de/html/ebm.php. Zugegriffen: 17. Sept. 2018

23. Kurch-Bek D, Sundmacher L, Gallowitz C, Tenckhoff B (2016) Vertragsärztliche Versorgung von Patienten in beschützenden Einrichtungen und Heimen. Analyse auf basis von Sekundärdaten (Treatment by panel physicians of patients in 
protective institutions and homes : analysis based on secondary data). Z Gerontol Geriatr. https://doi. org/10.1007/s00391-016-1144-8

24. Bundesinstitut für Bau-, Stadt- und Raumforschung (BBSR) (2009) Laufende Raumbeobachtung - Raumabgrenzungen. Siedlungsstrukturelle Kreistypen. http://www.bbsr.bund.de/BBSR/ DE/Raumbeobachtung/Raumabgrenzungen/ SiedlungsstrukturelleGebietstypen/Kreistypen/ kreistypen.html.Zugegriffen: 24. Febr. 2017

25. Statistisches Bundesamt (2018) Pflegeheime und verfügbare Plätze in Pflegeheimen.http://www.gbe-bund.de/oowa921-install/ servlet/oowa/aw92/dboowasys921.xwdevkit/ xwd_init?gbe.isgbetol/xs_start_neu/\&p_aid=i\& p_aid $=9189281 \&$ nummer $=570 \& p \_$sprache $=D \&$ p_indsp=108\&p_aid=6857821. Zugegriffen: 4 . Okt. 2018

26. Robert Koch-Institut (RKI) (2014) Beiträge zur Gesundheitsberichterstattung des Bundes. https://www.rki.de/DE/Content/ Gesundheitsmonitoring/Gesundheits berichterstattung/GBEDownloadsB/ GEDA12.pdf?_blob=publicationFile. Zugegriffen: 24. Sept. 2018

27. Bodeker B, Remschmidt C, Muters S, Wichmann O (2015) Influenza, tetanus, and pertussis vaccination coverage among adults in Germany (Impfquoten unter Erwachsenen in Deutschland fur die Impfungen gegen saisonale Influenza, Tetanus und Pertussis). Bundesgesundheitsblatt Gesundheitsforschung Gesundheitsschutz 58(2):174-181. https://doi.org/10.1007/s00103014-2097-y

28. Ljungman P, Cordonnier C, Einsele H et al (2009) Vaccination of hematopoietic cell transplant recipients. Bone Marrow Transplant 44(8):521-526. https://doi.org/10.1038/bmt.2009.263

29. Robert Koch-Institut (RKI) Saisonale Influenzaimpfung: Häufig gestellte Fragen und Antworten. https://www.rki.de/SharedDocs/FAQ/Impfen/ Influenza/faq_ges.html\#FAQId6948464.Zugegriffen: $10.0 k t .2018$

30. Reckendrees B, Mertens B, Wortberg S, Gaczkowska A, Stander V (2013) Einstellungen, Wissen und Verhalten der Allgemeinbevölkerung zu Hygiene und Infektionsschutz. Bundeszentrale für gesundheitliche Aufklärung (BZgA). http://epflicht.ulb. uni-bonn.de/content/titleinfo/248833. Zugegriffen:20.Dez. 2017

31. Robert Koch-Institut (RKI) (2017) Epidemiologisches Bulletin des Robert Koch-Instituts (RKI) Ausgabe 34/2017. https://www.rki.de/DE/ Content/Infekt/EpidBull/Archiv/2017/Ausgaben/ 34_17.pdf?_blob=publicationFile. Zugegriffen: 21. Sept. $201 \overline{18}$

32. Reuss AM, Walter D, Feig M et al (2010) Influenza vaccination coverage in the 2004/05, 2005/06, and 2006/07 seasons: a secondary data analysis based on billing data of the German associations of statutory health insurance physicians. Dtsch Arztebl Int 107(48):845-850. https://doi.org/10. 3238/arztebl.2010.0845

33. Robert Koch-Institut (RKI) (2017) Epidemiologisches Bulletin 1/2017. https://www.rki.de/DE/ Content/Infekt/EpidBull/Archiv/2017/Ausgaben/ 01_17.pdf?_blob=publicationFile. Zugegriffen: 14. Sept. 2018

34. Mereckiene J (2015) Seasonal influenza vaccination in Europe. Overview of vaccination recommendations and coverage rates in the EU member states for the 2012-13 influenza season. http://ecdc.europa.eu/en/publications/
Publications/Seasonal-influenza-vaccinationEurope-2012-13.pdf.Zugegriffen: 13. Febr. 2017

35. Centers for Disease Control and Prevention (2016) Flu vaccination coverage 2015-16

36. Vaux S, Noel D, Fonteneau L, Guthmann J-P, LevyBruhl D (2010) Influenza vaccination coverage of healthcare workers and residents and their determinants in nursing homes for elderly people in France: a cross-sectional survey. BMC Public Health 10:159. https://doi.org/10.1186/14712458-10-159

37. de Souto Barreto P, Lapeyre-Mestre M, Vellas $B$, Rolland Y (2014) Indicators of influenza and pneumococcal vaccination in French nursing home residents in 2011. Vaccine 32(7):846-851. https://doi.org/10.1016/j.vaccine.2013.12.023

38. Li Y, MukamelDB (2010) Racial disparities in receipt of influenza and pneumococcus vaccinations among US nursing-home residents. Am J Public Health 100(Suppl 1):62. https://doi.org/10.2105/ AJPH.2009.173468

39. European Centre for Disease Prevention and Control. Seasonal influenza vaccination in Europe. Vaccination recommendations and coverage rates in the EU Member States for eight influenza seasons: 2007-2008 to 2014-2015. Stockholm: ECDC; 2017 